\title{
Measurements of the production of prompt photons, jets and vector bosons+jets in pp collisions with the ATLAS detector
}

\author{
Marisilvia Donadelli, for the ATLAS Collaboration, a \\ ${ }^{1}$ University of Sao Paulo, Institute of Physics, 05508-090 - Sao Paulo - Brazil
}

\begin{abstract}
The production of jets, photons and gauge bosons in association with jets at hadron colliders provides a stringent test of perturbative QCD and can be used to probe the gluon density function of the proton. In this letter we present recent measurements of the inclusive jet cross section in data collected at center-of-mass energies of $8 \mathrm{TeV}$ and $13 \mathrm{TeV}$. The measurements have been performed differentially in various kinematic and angular observables and are compared with state-of-the-art predictions at NLO in $\mathrm{pQCD}$. ATLAS has performed precise measurements of the inclusive production of isolated prompt photons in data collected at center-of-mass energies of 8 and $13 \mathrm{TeV}$, differential in both rapidity and the photon transverse momentum. The measurements are compared with NLO pQCD calculations and with several MC generators. The cross section of photons, $W$ and $Z$ bosons in association with one or more jets have been measured differentially in various observables at center-of-mass energies of 8 and $13 \mathrm{TeV}$ and compared with state-of-the-art pQCD calculations up to NNLO QCD and NLO EWK as well as LO and NLO ME+PS generators. Dedicated phase spaces sensitive to emission of massive bosons collinear with jets or the electroweak production of vector bosons are tested.
\end{abstract}

\section{Introduction}

With the large data sample accumulated at the Large Hadron Collider (LHC) during the Run-1 and Run-2 periods at center-of-mass energies of $8 \mathrm{TeV}$ and $13 \mathrm{TeV}$, respectively, detailed investigations of perturbative quantum chromodynamics (pQCD) and electroweak (EWK) effects were conducted by the ATLAS experiment [1]. Predictions from different Monte Carlo generators based on leading-order (LO) and next-to-leading-order (NLO) matrix elements interfaced with parton shower and hadronisation models, and fixed-order predictions at next-to-leading order (NLO) and next-to-next-to-leading order (NNLO) are compared to the observed cross sections presented differentially in various kinematic and topological observables for the production of jets, photons and vector bosons in association with jets. The Monte Carlo simulations are also used to estimate most of the contributions from background events and to unfold the data to the particle level. The unfolding procedure for all the results in the next sections follow the Bayesian Iterative method [2], as implemented in the RooUnfold package [3].

\footnotetext{
a e-mail: marisilvia.donadelli@ cern.ch
} 


\section{Production of Jets}

At high transverse momenta the scattering of partons can be calculated using pQCD and jet measurements are directly related to the scattering of quarks and gluons. The large cross sections for such processes allow for differential measurements in a wide kinematic range and stringent test of the underlying theory. Jets are reconstructed with the anti- $k_{t}$ algorithm [4] with four-momentum recombination as implemented in the FastJet package [5], with radius parameter $R=0.4$. The jet differential cross section results, as a function of a variety of kinematic and topological variables, are shown with the corresponding distributions presented in the analyses, which are individually unfolded to deconvolve detector effects such as inefficiencies and resolutions [6].

\subsection{Four-jet production at $8 \mathrm{TeV}$}

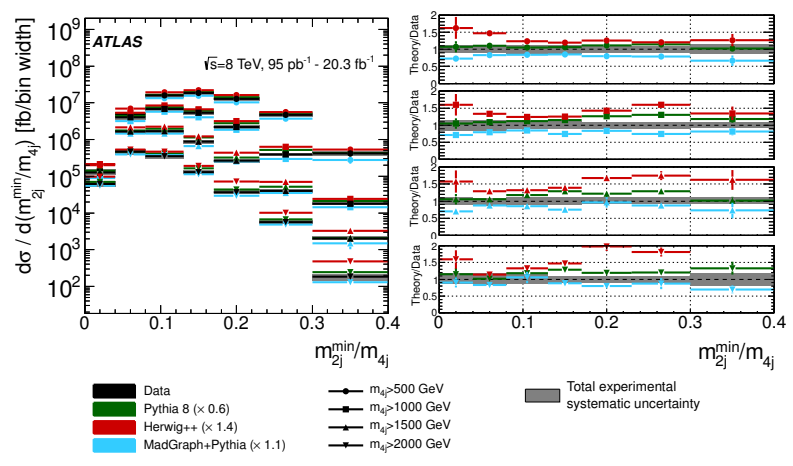

Figure 1. Unfolded four-jet differential cross section as a function of $m_{2 \mathrm{j}}^{\min } / m_{4 \mathrm{j}}$, compared to different theoretical predictions: Pythia, Herwig++ and MadGraph+Pythia [6].

With a dataset corresponding to an integrated luminosity of $20.3 \mathrm{fb}^{-1}$, the differential cross sections for events with at least four jets were studied as a function of momentum, masses and angles [6]. The cross sections are calculated for events with at least four jets, within the rapidity range $|y|<2.8$. Out of those four jets, the leading one must have $p_{\mathrm{T}}>100 \mathrm{GeV}$, while the next three must have $p_{\mathrm{T}}>64 \mathrm{GeV}$, with the condition that these four jets must be well separated from one another by $\Delta R_{\min }>0.65$. Considering that mass variables are widely used in physics searches, and that they are also sensitive to events with large separations between jets, Figure 1 shows the unfolded distribution of the minimum invariant mass of two jets relative to the invariant mass of four jets, along-

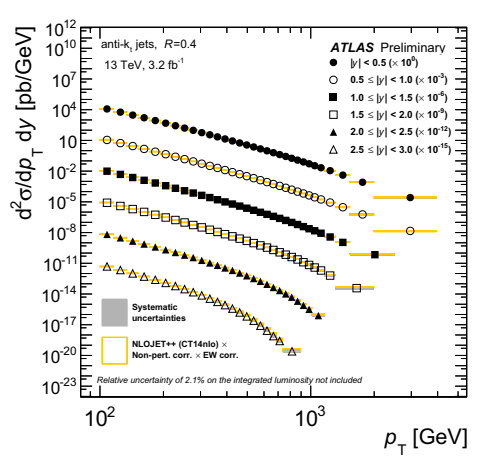

Figure 2. Inclusive-jet cross-sections as a function of $p_{\mathrm{T}}$ and $|y|$, for anti- $k_{t}$ jets with $\mathrm{R}=0.4$ [7]. 
side with the generators used for comparison against data [6]. One can see that MadGraph+Pythia provides a very good description, with a flat ratio for all the $m_{4 \mathrm{j}}$ cuts.

\subsection{Inclusive jets at $13 \mathrm{TeV}$}

Inclusive-jet cross-sections are measured using data corresponding to an integrated luminosity of 3.2 $\mathrm{fb}^{-1}$ [7]. The jet cross-sections are defined using jets representing those clustered from stable particles with a proper mean lifetime $(\tau)$ exceeding $10 \mathrm{~mm}$, with muons and neutrinos from decaying hadrons included in the jet clustering. Figure 2 shows the inclusive-jet cross-section result for six jet rapidity intervals covering the jet $p_{\mathrm{T}}^{\text {jet }}$ range from $100 \mathrm{GeV}$ to $\sim 3.2 \mathrm{TeV}$ and within a jet rapidity interval $|y|<3$, together with the NLO pQCD predictions calculated using NLOJET++ 4.1.3 [8] with the CT14 NLO PDF set [9], to which non-perturbative and electroweak corrections are applied. The dominant systematic uncertainty for this result, which significantly extends the reach of ATLAS previous measurement [10], arises from the jet energy calibration [7].

\section{Isolated prompt photons at $8 \mathrm{TeV}$}

The production of prompt photons provides a probe of $\mathrm{pQCD}$ in a cleaner environment compared to jet production and moreover, through the dominant LO process $q g \rightarrow q \gamma$, can be used to study the gluon parton distribution function of the proton [11-15]. At LO in pQCD, two processes contribute to prompt photon production: the direct-photon process, in which the photon originates directly from the hard interaction, and the fragmentation-photon process, in which the photon is emitted in the fragmentation of a high transverse momentum parton.

With an integrated luminosity of $20.2 \mathrm{fb}^{-1}$, the differential cross section of inclusive photon production was measured as a function of $E_{\mathrm{T}}^{\gamma}$, split in 4 bins of pseudorapidity covering the range $\left|\eta^{\gamma}\right|<1.37$ and $1.56<\left|\eta^{\gamma}\right|<2.37$, spanning the photon transverse energy between 25 and 1500 $\mathrm{GeV}$ [16]. The photon candidate, identified by combining the information from the calorimeters and the inner tracker, is required to be isolated based on the amount of transverse energy inside a cone of $\Delta R=0.4$ in the $\eta-\phi$ plane centred around the photon direction, to avoid the large contribution of photons from neutral-hadron decays. The theoretical calculations used in the analysis consist of LO Monte Carlo event generators and calculations at NLO or higher. JetPhox is used as the baseline to compare the results as it is capable of calculating the double-differential inclusive prompt photon cross section at parton level to NLO accuracy for both the direct and fragmentation photon processes. PeTeR [17] is used as a second parton-level generator to predict the differential isolated prompt photon cross section at NLO including the resummation of threshold logarithms at the next-to-nextto-next-to-leading- logarithmic (NNNLL) level. It can be seen in Figure 3 that PeTeR removes the normalisation difference seen between data and JetPhox, especially in the region $|\eta|<1.37$. The uncertainties shown, from combining the scale, PDF and electroweak uncertainties, are about $20 \%$ lower than those from JetPhox. The PeTeR predictions match the data, within the combined measured and theoretical uncertainties, in all of the measured phase space [16].

\section{Vector bosons + jets production}

The relatively large production cross sections and easily identifiable decays of the boson to charged leptonic final states offer clean experimental signatures which can be precisely measured. Such processes also constitute a non-negligible background for Higgs boson studies and in searches for new phenomena. Typically in these studies, the multiplicity and kinematics of the jets are exploited to achieve a separation of the signal of interest from the Standard Model (SM) vector boson + jets process. 

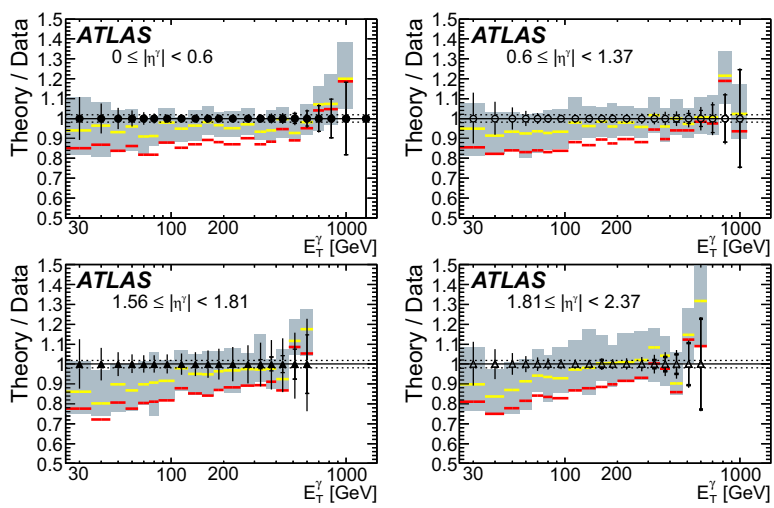

\section{ATLAS}

$\sqrt{\mathrm{s}}=8 \mathrm{TeV}, 20.2 \mathrm{fb}^{-1}$

Data 2012

- $0 \leq\left|\eta^{\gamma}\right|<0.6$

$0.6 \leq\left|\eta^{\gamma}\right|<1.37$

$\Delta 1.56 \leq\left|\eta^{\top}\right|<1.81$

$\Delta 1.81 \leq\left|\eta^{\gamma}\right|<2.37$

- Lumi Uncert.

NLO:

PETER CT10

- JetPhox CT10

Figure 3. Ratio of theory (PeTeR and JetPhox both using the CT10 PDF) to data for the differential cross sections as a function of $E_{\mathrm{T}}^{\gamma}$ for the four $\eta^{\gamma}$ regions [16].

The measurements were compared to fixed-order calculations at NLO BlackHat+Sherpa $[19,20]$ and at NNLO from $Z+\geq 1$ jet $N_{\text {jetti }}$ and $W+\geq 1$ jet $N_{\text {jetti }}$ [21-23] which use a new subtraction technique based on $N$ - jettiness [24] and relies on the theoretical formalism provided in soft-collinear effective theory. The measured cross sections are also compared to predictions from the generators Alpgen v2.14 [25] interfaced to Pythia v6.426 [26] to model the parton shower (PS), denoted as Alpgen+Py6, and to Madgraph MG5_aMC NLO v2.2.2 generator [27], in two different schemes: MG5_aMC+Py8 CKKWL, using matrix elements including up to four partons at leading order, interfaced to Pythia v8.186 [28], using the CKKWL merging scheme [29] and with ME for up to two jets at NLO and with parton showers beyond this generated using the FxFx scheme [30]. The cross sections results are also compared to $W+\mathrm{j}$ and $W+\mathrm{j} \mathrm{j}+$ weak shower calculation provided by Pythia v8.210 and, in this case, the $W$ boson can either be produced by the matrix elements of the $W+1$ jet final state or be emitted as electroweak finalstate radiation in the parton shower of a dijet event. The Sherpa + OpenLoops $W+\mathrm{j}$ and $W+\mathrm{j} \mathrm{j}$ calculation incorporates NLO QCD and NLO EW corrections to both of these processes [31-35].

\subsection{Z+jets at $13 \mathrm{TeV}$}

Measurements of the cross section for the production of a $\mathrm{Z}$ boson decaying to electrons or muons associated with up to seven jets in the jet kinematic range of transverse momentum $p_{\mathrm{T}}>30 \mathrm{GeV}$ and rapidity $|y|<2.5$ were performed using data corresponding to an integrated luminosity of $3.16 \mathrm{fb}^{-1}$. The invariant mass of the lepton pair was selected within $71<m_{\ell \ell}<111 \mathrm{GeV}$ and the jet and the lepton were required to have a separation of $\Delta R(\ell$, jet $)>0.4$ [18].

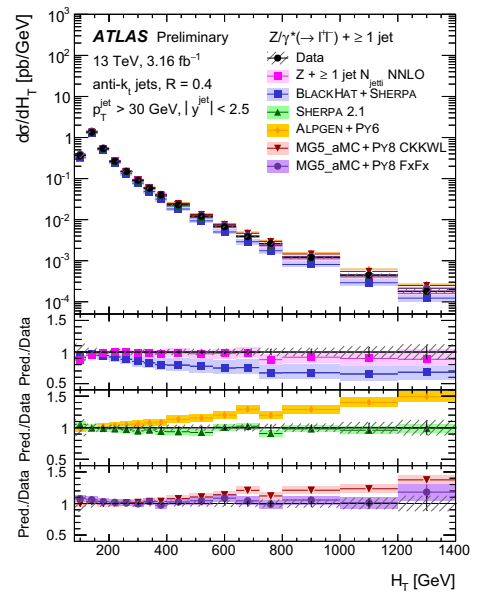

Figure 4. Measured cross section as a function of the $H_{\mathrm{T}}$ for inclusive $Z+\geq 1$ jet events [18]. 
Figure 4 shows the measured cross sections as a function of the $H_{\mathrm{T}}$ distribution (up to $1400 \mathrm{GeV}$ ) in inclusive $Z+\geq 1$ jet events. Sherpa 2.1 [36] and MG5_aMC+Py8 FxFx [30] describe well the spectrum while MG5_aMC+Py8 CKKWL [29] and Alpgen+Py6 overestimate the distribution at large values of $H_{\mathrm{T}}$. BlackHat+Sherpa under-estimates the cross section for $H_{\mathrm{T}}>300 \mathrm{GeV}$, as observed in similar measurements at lower centre-of-mass energies, due to the missing contributions from events with higher parton multiplicities. One can note that the agreement is recovered by adding higher orders in perturbative QCD, as demonstrated by the good description of $H_{\mathrm{T}}$ by $\mathrm{N}_{\text {jetti }} \mathrm{NNLO}$ [18].

\subsection{Collinear W production at $8 \mathrm{TeV}$}

The $W$ boson angular distribution in events with high transverse momentum jets was measured using data corresponding to an integrated luminosity of $20.3 \mathrm{fb}^{-1}$ [37]. The focus is on the contributions to $W+$ jets processes from real $W$ emission, so that the selected events contain an isolated muon and a jet with transverse momentum $p_{\mathrm{T}}>500 \mathrm{GeV}$. In this kinematic regime, contributions to $W+$ jets processes from real $\mathrm{W}$ boson emission are enhanced in the region of small angular separation between the $W$ boson decay products and the closest jet, with the angular separation defined as the distance between the muon and the closest jet, $\Delta R(\mu$, jet $)=$

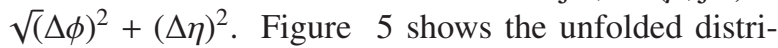
bution from background-subtracted data of the angular separation between the muon and the closest jet in the signal region for events with $500 \mathrm{GeV}<p_{\mathrm{T}}$ (leading jet) $<600 \mathrm{GeV}$ [37] along with a variety of MC generators and theoretical calculations. Alpgen+Pythia6 overestimates the total cross-section, whereas Pythia8, which is modified to explicitly include the process of $\mathrm{W}$ boson emission, disagrees with the measurement in the collinear region $(\Delta R(\mu$, jet $)<2.4)$. Agreement with the Sherpa+OpenLoops NLO QCD+EW calculation and the $W+\geq 1$ jet $\mathrm{N}_{\text {jetti }}$ calculation [23] is well within the systematic and statistical uncertainties of the predictions and the measurement.

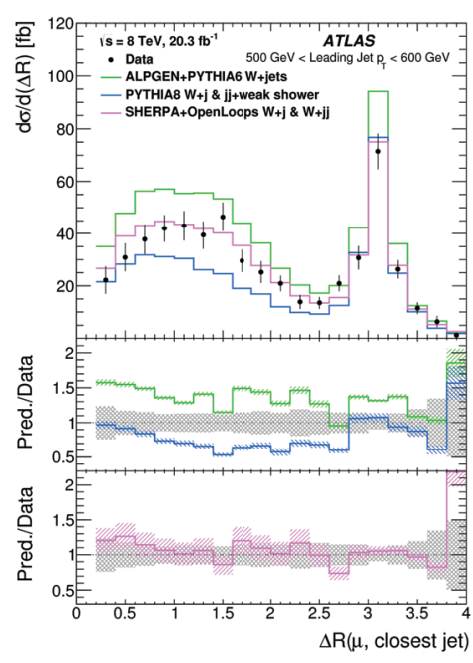

Figure 5. Unfolded distribution from background-subtracted data of the angular separation between the muon and the closest jet in the signal region along with several predictions from theory calculations for events with $500 \mathrm{GeV}<$ leading jet $p_{\mathrm{T}}<600 \mathrm{GeV}$ [37].

\section{Conclusions}

Measurements of the production of jets, photons and vector bosons plus jets were presented in various kinematic and topological observables and compared to predictions from different Monte Carlo generators. MadGraph+Pythia provides the best description of mass variables for the four-jet results at $8 \mathrm{TeV}$. NLO pQCD with non-perturbative and electroweak effects corrections are compared to the inclusive jets measurement at $13 \mathrm{TeV}$. The predictions are in good agreement with data, confirming the validity of $\mathrm{pQCD}$ in the measured kinematic regions. The isolated prompt photons result covers ten orders of magnitude in cross section, with NLO QCD (PeTeR) with resummation+electroweak 
corrections describing data well within uncertainties. Vector boson+jets results provide essential input for optimisation of the Monte Carlo generators and, in particular, the collinear $W$ measurement has implications for Monte Carlo programs that incorporate real $W$ boson emission, a process which is only just now being probed directly at the energy of the LHC.

\section{References}

[1] G. Aad et al. (ATLAS), Journal of Instrumentation 3, S08003 (2008)

[2] G. D’Agostini, Nucl. Instrum. Meth. A362, 487 (1995)

[3] T. Adye, Unfolding algorithms and tests using RooUnfold (2011), 1105.1160

[4] M. Cacciari et al., JHEP 04, 063 (2008), 0802 . 1189

[5] M. Cacciari et al., Eur. Phys. J. C72, 1896 (2012), 1111.6097

[6] G. Aad et al. (ATLAS), JHEP 12, 105 (2015), 1509. 07335

[7] Tech. Rep. ATLAS-CONF-2016-092 (2016), http://cds . cern. ch/record/2209210

[8] Z. Nagy, Phys. Rev. D68, 094002 (2003), hep-ph/0307268

[9] S. Dulat et al., Phys. Rev. D93, 033006 (2016), 1506.07443

[10] G. Aad et al. (ATLAS), JHEP 02, 153 (2015), [Erratum: JHEP09,141(2015)], 1410. 8857

[11] H.L. Lai et al., Phys. Rev. D51, 4763 (1995), hep-ph/9410404

[12] A.D. Martin et al., Eur. Phys. J. C4, 463 (1998), hep-ph/9803445

[13] A.D. Martin et al., Eur. Phys. J. C14, 133 (2000), hep-ph/9907231

[14] P. Aurenche et al., Phys. Rev. D73, 094007 (2006), hep-ph/0602133

[15] D. d'Enterria, J. Rojo, Nucl. Phys. B860, 311 (2012), 1202 . 1762

[16] G. Aad et al. (ATLAS), JHEP 08, 005 (2016), 1605.03495

[17] T. Becher, C. Lorentzen, M.D. Schwartz, Phys. Rev. D86, 054026 (2012), 1206.6115

[18] Tech. Rep. ATLAS-CONF-2016-046 (2016), https://cds. cern. ch/record/2206128

[19] C.F. Berger et al., Phys. Rev. D82, 074002 (2010), 1004.1659

[20] H. Ita et al., Phys. Rev. D85, 031501 (2012), 1108. 2229

[21] R. Boughezal et al., Phys. Rev. Lett. 116, 152001 (2016), 1512.01291

[22] R. Boughezal et al., Phys. Rev. D94, 074015 (2016), 1602.08140

[23] R. Boughezal et al. (2016), 1602.06965

[24] J. Gaunt et al., JHEP 09, 058 (2015), 1505 . 04794

[25] M.L. Mangano et al., JHEP 07, 001 (2003), hep-ph/0206293

[26] T. Sjostrand et al., JHEP 05, 026 (2006), hep-ph/0603175

[27] J. Alwall et al., JHEP 07, 079 (2014), 1405.0301

[28] T. Sjostrand et al., Comput. Phys. Commun. 178, 852 (2008), 0710. 3820

[29] L. Lonnblad, JHEP 05, 046 (2002), hep-ph/0112284

[30] R. Frederix et al., JHEP 02, 131 (2016), 1511 . 00847

[31] S. Kallweit et al., JHEP 04, 021 (2016), 1511.08692

[32] F. Krauss et al., JHEP 02, 044 (2002), hep-ph/0109036

[33] T. Gleisberg et al., Eur. Phys. J. C53, 501 (2008), 0709. 2881

[34] F. Cascioli et al., Phys. Rev. Lett. 108, 111601 (2012), 1111. 5206

[35] A. Denner et al., PoS LL2014, 071 (2014), 1407.0087

[36] T. Gleisberg et al., JHEP 02, 007 (2009), 0811. 4622

[37] M. Aaboud et al. (ATLAS) (2016), 1609.07045 